\title{
Histone deacetylases 1 and 2 redundantly regulate cardiac morphogenesis, growth, and contractility
}

\author{
Rusty L. Montgomery, ${ }^{1,4}$ Christopher A. Davis, ${ }^{1,4}$ Matthew J. Potthoff, ${ }^{1}$ Michael Haberland, ${ }^{1}$ \\ Jens Fielitz, ${ }^{1}$ Xiaoxia Qi, ${ }^{1}$ Joseph A. Hill, ${ }^{2}$ James A. Richardson, ${ }^{1,3}$ and Eric N. Olson ${ }^{1,5}$ \\ ${ }^{1}$ Department of Molecular Biology, The University of Texas Southwestern Medical Center at Dallas, Dallas, \\ Texas 75390, USA; ${ }^{2}$ Department of Internal Medicine, The University of Texas Southwestern Medical Center at Dallas, \\ Dallas, Texas 75390, USA; ${ }^{3}$ Department of Pathology, The University of Texas Southwestern Medical Center at Dallas, \\ Dallas, Texas 75390, USA
}

\begin{abstract}
Histone deacetylases (HDACs) tighten chromatin structure and repress gene expression through the removal of acetyl groups from histone tails. The class I HDACs, HDAC1 and HDAC2, are expressed ubiquitously, but their potential roles in tissue-specific gene expression and organogenesis have not been defined. To explore the functions of HDAC1 and HDAC2 in vivo, we generated mice with conditional null alleles of both genes. Whereas global deletion of HDAC1 results in death by embryonic day 9.5, mice lacking HDAC2 survive until the perinatal period, when they succumb to a spectrum of cardiac defects, including obliteration of the lumen of the right ventricle, excessive hyperplasia and apoptosis of cardiomyocytes, and bradycardia. Cardiac-specific deletion of either HDAC1 or HDAC2 does not evoke a phenotype, whereas cardiac-specific deletion of both genes results in neonatal lethality, accompanied by cardiac arrhythmias, dilated cardiomyopathy, and up-regulation of genes encoding skeletal muscle-specific contractile proteins and calcium channels. Our results reveal cell-autonomous and non-cell-autonomous functions for HDAC1 and HDAC2 in the control of myocardial growth, morphogenesis, and contractility, which reflect partially redundant roles of these enzymes in tissue-specific transcriptional repression.
\end{abstract}

[Keywords: Heart development; histone deacetylase; transcription]

Supplemental material is available at http://www.genesdev.org.

Received April 20, 2007; revised version accepted June 13, 2007.

Chromatin-modifying enzymes control gene expression through reversible post-translational modifications of histone tails within nucleosomes (Jenuwein and Allis 2001). Histone acetyltransferases catalyze the transfer of acetyl groups from acetyl coenzyme A to $\varepsilon$-amino groups of lysine residues of histone tails. Acetylation results in a loss of positive charge, relaxing chromatin structure and allowing access of transcription factors to their target genes (Roth et al. 2001). Histone deacetylases (HDACs) oppose the activity of histone acetyltransferases by catalyzing the removal of acetyl groups from histone tails, resulting in compaction of chromatin and transcriptional repression (Grozinger and Schreiber 2002).

There are four classes of HDACs that comprise an ancient enzyme family conserved from bacteria to humans (Gregoretti et al. 2004). Class I HDACs (HDAC1, HDAC2, HDAC3, and HDAC8) are highly homologous to the yeast HDAC RPD3 and are ubiquitously expressed (Yang

\footnotetext{
${ }^{4}$ These authors contributed equally to this work.

${ }^{5}$ Corresponding author.

E-MAIL Eric.Olson@utsouthwestern.edu; FAX (214) 648-1196.

Article is online at http://www.genesdev.org/cgi/doi/10.1101/gad.1563807.
}

et al. 1997). The class II HDACs (HDAC4, HDAC5, HDAC7, and HDAC9) are homologous to the yeast protein HDA1 and are enriched in muscle and neural tissues (Grozinger et al. 1999; Verdin et al. 2003). Class II HDACs regulate tissue growth through signal-dependent repression of MEF2 and other transcription factors (McKinsey et al. 2000). Class III HDACs, or sirtuins, require NAD for deacetylation and are related to the yeast repressor Sir2 (Grozinger and Schreiber 2002). Class IV HDACs, a recently identified family of deacetylases, share homology with human HDAC11 (Gao et al. 2002).

The functions of class I HDACs in mammals have been largely inferred from studies in cultured cells, but relatively little is known of their potential roles in vivo. HDAC1 and HDAC2 share $85 \%$ amino acid homology and are found together in almost all repressive transcriptional complexes (Grozinger and Schreiber 2002). Mouse embryos deficient in HDAC1 die before embryonic day 10.5 (E10.5) due to proliferative defects (Lagger et al. 2002 ), precluding an analysis of its potential functions later in development or after birth. An HDAC2 mutant mouse generated from a lacZ insertion was recently reported to be viable (Trivedi et al. 2007), although as dis- 
cussed below, our findings differ from the conclusions of that study.

To examine the functions of HDAC1 and HDAC2 in vivo, we generated mice with conditional null alleles for both genes. We show that global deletion of HDAC2 results in perinatal lethality with severe cardiac defects that appear to reflect a non-myocyte-autonomous function of HDAC2, since cardiac-specific deletion of either HDAC1 or HDAC2 alone has no discernable effect on cardiac function. However, cardiac deletion of both HDAC1 and HDAC2 genes results in dilated cardiomyopathy, arrhythmias, and neonatal lethality, accompanied by up-regulation of genes encoding skeletal musclespecific myofibrillar proteins and calcium channels. Our findings suggest that HDAC1 and HDAC2 are functionally redundant in cardiac growth and development and maintain cardiomyocyte identity and function, at least in part, through repression of genes encoding skeletal musclespecific myofibrillar proteins and calcium channels.

\section{Results}

\section{Conditional deletion of HDAC1}

The mouse HDAC1 gene contains 14 exons and spans 30 $\mathrm{kb}$ of DNA (Khier et al. 1999). We generated a conditional null allele of HDAC1 by introducing lox $P$ sites upstream of exon 5 and downstream from exon 7 through homologous recombination in embryonic stem (ES) cells (Fig. 1). This deletion eliminates several key histidine and aspartic acid residues required for catalytic activity (Hassig et al. 1998). Mice heterozygous for this $H D A C 1^{\text {neo-loxP }}$ allele were bred to females expressing a CAG-Cre transgene, which deletes in the germline (Sakai and Miyazaki 1997), resulting in an $H D A C 1^{+/-}$ allele. $H D A C 1^{+/-}$mice were intercrossed to generate $H D A C 1^{-/-}$mice on a $129 / \mathrm{C} 57 \mathrm{BL} / 6 / \mathrm{CD} 1$ mixed genetic background. Homozygosity of the HDAC1-null allele resulted in embryonic lethality before E9.5, consistent with a prior description of the global HDAC1 gene deletion (Fig. 1D; Lagger et al. 2002).

$H D A C 1^{\text {neo-loxP }}$ mice were bred to FLPe transgenic animals to delete the neomycin cassette (Rodriguez et al. 2000), resulting in an $H D A C 1^{\text {loxP }}$ allele (Fig. 1A). We deleted HDAC1 specifically in the heart by breeding homozygous $H D A C 1^{\text {loxP/loxP }}$ mice to transgenic mice expressing Cre recombinase under the control of the $\alpha$-myosin heavy chain $(\alpha M H C)$ promoter, which is expressed in differentiated cardiomyocytes pre- and postnatally (Agah et al. 1997). HDAC1 $1^{10 x P / / o x P} ; \alpha M H C$-Cre mice were phenotypically normal and displayed no cardiac abnormalities (Fig. 2A). HDAC1 immunostaining of cardiomyocytes isolated from $H D A C 1^{\operatorname{lox} P / \operatorname{lox} P} ; \alpha M H C$ -

A

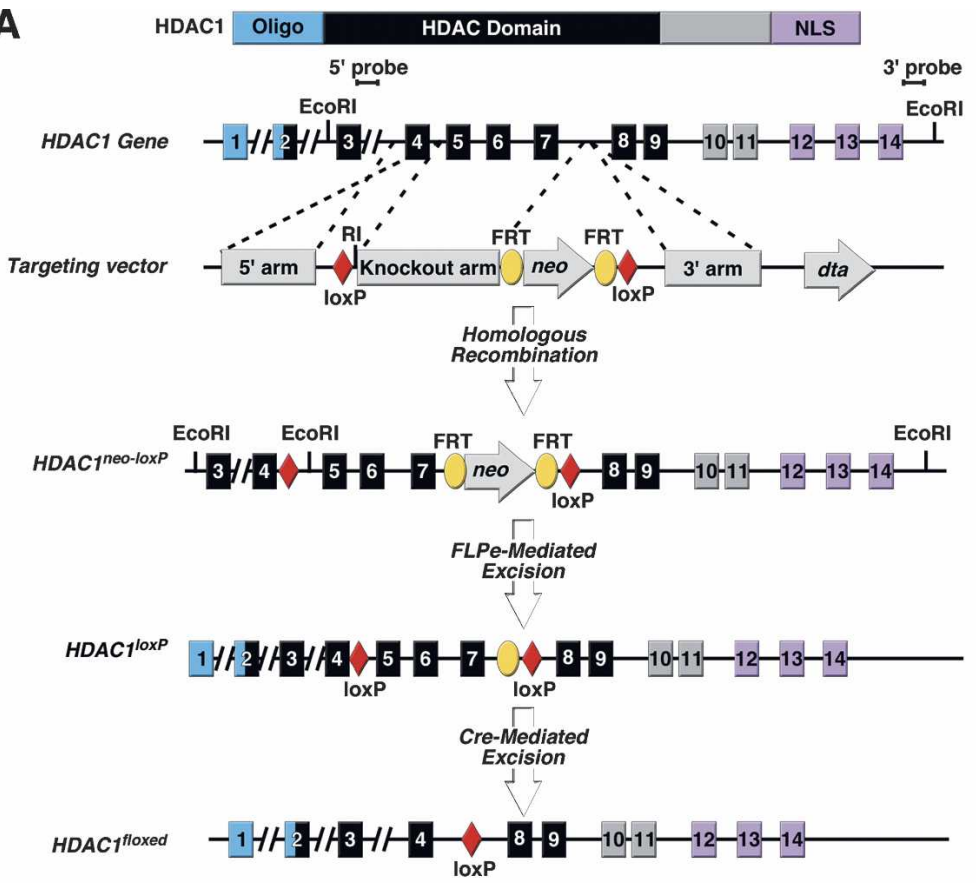

B

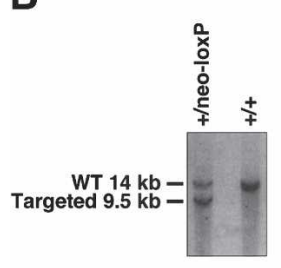

C

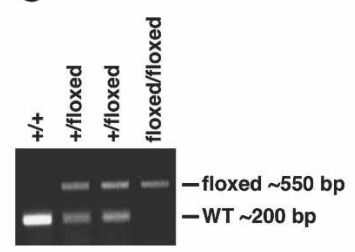

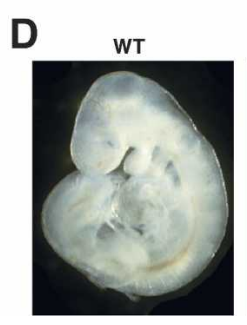

Figure 1. Generation of a conditional $H D A C 1$ allele. $(A)$ Strategy to generate a conditional HDAC1 allele. Protein, corresponding exonic structure, targeting vector, and targeted allele are shown. $\operatorname{lox} P$ sites were inserted into introns 4 and 7 through homologous recombination. The neomycin resistance cassette, flanked by FRT sites, was removed by crossing to transgenic animals expressing hACTB::FLPe in the germline. Cre-mediated excision results in one $\operatorname{lox} P$ site in the place of exons 5-7. (B) Southern blot analysis of agouti offspring from chimera/ C57BL/6 intercrosses from targeted ES cells. Tail DNA was digested with EcoRI, and the corresponding wild-type $(\sim 14 \mathrm{~kb})$ and targeted $(\sim 9.5 \mathrm{~kb})$ bands are indicated for the 3' probe. (C) Genotyping of $H D A C 1^{\text {floxed }}$ mice by genomic PCR. Primer set includes three primers. One primer set flanks the $5^{\prime} \operatorname{lox} P$ site with the third primer downstream from the $3^{\prime}$ lox $P$ site. Global deletion by CAG-Cre removes the primer within the $\operatorname{lox} P$ sites, resulting in one $\sim 550-\mathrm{bp}$ product for the $H D A C 1^{\text {floxed/floxed }}$ animals. $(D)$ Wild-type and $H D A C 1^{\operatorname{lox} P / / \operatorname{lox} P} ; C A G$-Cre mutant embryos at E9.5. Recapitulation of the previously reported global KO is shown. 
A

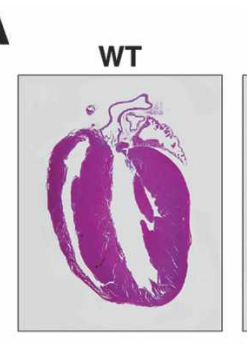
HDAC1loxP/loxP $\alpha M H C-C r$
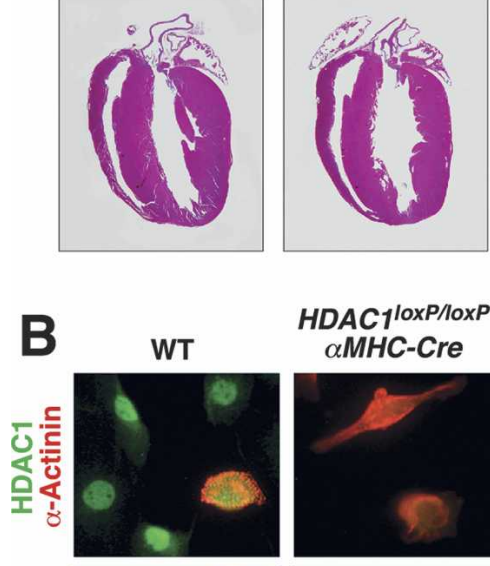

HDAC1loxP/loxP $\alpha M H C-C r e$
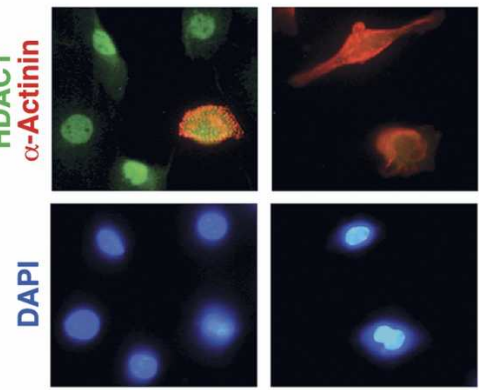

C

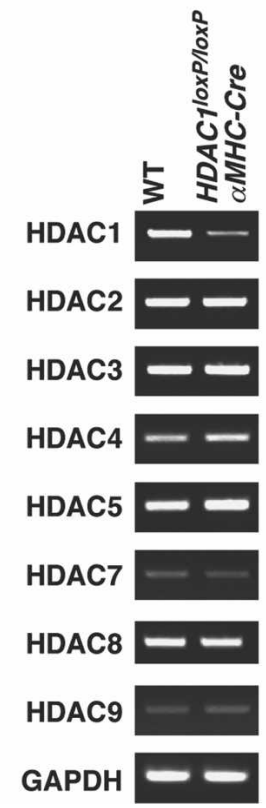

Figure 2. Cardiac-specific deletion of HDAC1. (A) Wild-type and $H D A C 1^{\operatorname{lox} P / \operatorname{lox} P} ; \alpha M H C$-Cre mice at $6 \mathrm{wk}$ of age. Cardiacspecific deletion of HDACl was achieved by crossing $H D A C 1^{10 x P / \operatorname{lox} P}$ mice to mice harboring a transgene for $\alpha \mathrm{MHC}$ Cre. Hematoxylin and eosin (H\&E)-stained hearts at 6 wk of age show no gross abnormalities. $(B)$ Immunostaining for HDAC1 (green) and $\alpha$-actinin (red) on cardiomyocytes isolated from wild-type and $H D A C 1^{\operatorname{lox} P / \operatorname{lox} P} ; \alpha M H C$-Cre neonatal mice. Note cardiac fibroblasts positive for HDAC1 that are negative for $\alpha$-actinin. Immunostaining for HDACl in Cre-positive myocytes shows no positive staining for HDAC1. (C) Transcript analysis of cardiac-specific deletion of HDAC1. Transcript levels were analyzed by semiquantitative RT-PCR for HDACs 1-5 and HDACs 7-9. GAPDH levels were detected as a control. HDAC1 transcript levels are not entirely lost due to high expression in contaminating cardiac fibroblasts, as seen in $B$.
Cre mice revealed no nuclear staining in cardiomyocytes positive for $\alpha$-actinin, confirming efficient recombination in vivo (Fig. 2B).

RT-PCR on RNA isolated from the hearts of $H D A C 1^{\operatorname{lox} P / \operatorname{lox} P} ; \alpha M H C$-Cre mice confirmed the reduction in expression of the HDAC1 transcript (Fig. 2C); residual transcript in the heart likely arises from fibroblasts and other nonmyocytes in which the $\alpha M H C$-Cre transgene is not expressed. Other class I HDAC levels were unchanged in the hearts of $H D A C 1^{\operatorname{lox} P / \operatorname{lox} P} ; \alpha M H C$ Cre mice (Fig. 2C), suggesting basal expression of other class I HDACs is sufficient to compensate for the loss of HDAC1. We also deleted HDAC1 in the neural crest, skeletal muscle, central nervous system, endothelium, smooth muscle, and secondary heart field using the appropriate Cre transgenic lines (Supplementary Table 1). All of these mutant mice were viable.

\section{Conditional deletion of HDAC2}

Given the persistent expression of other class I HDACs in the hearts of $H D A C 1^{\operatorname{lox} P / 10 x P} ; \alpha M H C$-Cre mice and the high homology between HDAC1 and HDAC2, we addressed the possible functional redundancy of these HDACs by generating a conditional allele for HDAC2. We introduced $\operatorname{lox} P$ sites upstream of exon 2 and downstream from exon 4 of the mouse HDAC2 gene, which contains 14 exons and spans $36 \mathrm{~kb}$ (Fig. 3A,B). This mutation deletes a portion of the oligomerization domain and residues in the catalytic domain required for enzymatic activity (Hassig et al. 1998). Mice heterozygous for the HDAC2 $2^{\text {neo-loxP }}$ allele were crossed to CAG-Cre transgenic mice (Sakai and Miyazaki 1997) to generate an $\mathrm{HDAC2}^{+/-}$allele, and $\mathrm{HDAC2}^{+/-}$mice were intercrossed to generate $H D A C 2^{-/-}$mice on a $129 / \mathrm{C} 57 \mathrm{BL} / 6 /$ CD1 mixed genetic background. $H D A C 2^{-/-}$mice were born at near expected Mendelian ratios; however, 100\% of null mice derived from two independent ES cell clones became cyanotic and died within the first $24 \mathrm{~h}$ after birth (Fig. 3C). At birth, HDAC2 ${ }^{-/-}$offspring were indistinguishable from wild-type littermates, but most failed to nurse and even those that did nurse succumbed within the same time period as mutants that lacked milk in their stomachs. The complete lethality of these HDAC2null mice contrasts with the conclusions of a recent study reporting that the majority of mice homozygous for a lacZ insertion mutation in $H D A C 2$, reputed to be a null allele, were viable (Trivedi et al. 2007).

RT-PCR using primers upstream of and downstream from the $\operatorname{lox} P$ sites showed a complete absence of HDAC2 mRNA encoding exons 2-4 in the homozygous HDAC2 mutant animals, whereas primers downstream from the loxP sites revealed nearly normal levels of transcript in the mutant mice (Fig. 3D). Sequencing of the mutant transcript showed two splice variants of the mutant HDAC2 allele, an in-frame transcript from exon 1 to exon 5 and an out-of-frame transcript from exon 1 to exon 6. However, we were unable to detect any expression of mutant HDAC2 protein from lung or heart tissue by Western blot using an HDAC2 antibody against the $\mathrm{C}$ 

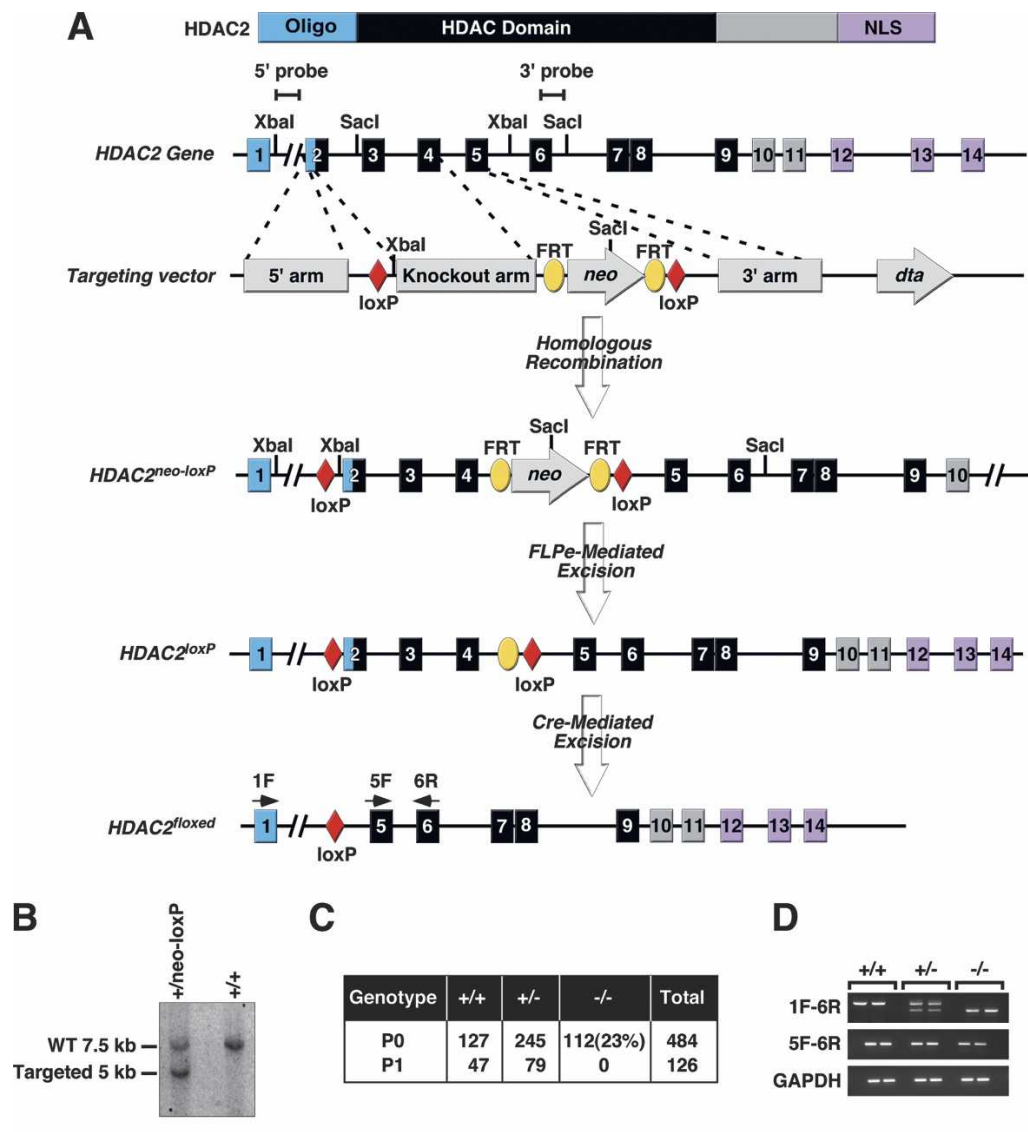

C

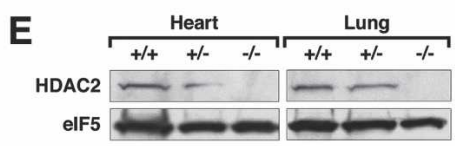

$$
\begin{aligned}
& \text { HDAC1 }+\frac{+1}{-1+} \\
& \text { HDAC3 }-- \\
& \text { HDAC4 }-- \\
& \text { HDAC5 }-- \\
& \text { HDAC7 }-- \\
& \text { HDAC8 }-- \\
& \text { HDAC9 }-- \\
& \text { GAPDH }--
\end{aligned}
$$

Figure 3. Generation of a conditional HDAC2 allele. (A) Strategy for targeting of HDAC2. Exonic structure, targeting vector, and targeted allele for HDAC2 are shown. $\operatorname{lox} P$ sites were introduced upstream of exon 2 and downstream from exon 4. Flanking FRT sites allowed for the neomycin resistance cassette to be removed by crossing to FLPe recombinase transgenic animals. Cre excision results in one $\operatorname{lox} P$ site in the place of exons 2-4. Probes for Southern analysis and primer positions for RT-PCR are shown. $(B)$ Southern blot analysis for HDAC2. Tail DNA from agouti offspring was digested with SacI and probed with the indicated $3^{\prime}$ probe. Wild-type $(7.5 \mathrm{~kb})$ and targeted $(5 \mathrm{~kb})$ bands indicate proper transmission of targeted allele. $(C)$ Table of offspring from HDAC2 heterozygous intercrosses. HDAC2-null mice were born at near Mendelian ratios, but showed $100 \%$ lethality within the first 24 h. (D) Transcript analysis of HDAC2 by RT-PCR. Genotypes of animals are shown at top, and primer positions from $A$ are shown to the left. GAPDH levels were detected as a control. $(E)$ Western blot analysis to show deletion of HDAC2. Western blot analysis on heart and lung tissue isolated from P1 wild-type, heterozygous, and HDAC2 homozygous-null neonates. eIF5 was detected as a loading control. $(F)$ Transcript analysis on HDAC2-null mice. mRNA transcript levels were analyzed by semiquantitative RTPCR for HDACs 1, 3-5, and 7-9. GAPDH was detected as a control. terminus (Fig. 3E), suggesting that the truncated mRNA is inefficiently translated or the truncated protein is unstable. Other class I and II HDACs were expressed at normal levels in the hearts of HDAC2-null mice (Fig. 3F).

\section{Cardiac abnormalities in HDAC2 mutant neonates}

Histological analysis of wild-type and $\mathrm{HDAC2}^{-/-}$neonates revealed no obvious defects in the brain, liver, lung, or skeletal muscle (data not shown). However, the hearts of mutant mice displayed unusual morphological abnormalities of the right ventricular chamber (Fig. 4A). In some HDAC2 mutant neonates, there was an almost complete loss of the right ventricular lumen, as revealed by serial sectioning of the heart. In other mutant hearts, we observed the right ventricle to be completely dorsal to the left ventricle. In addition, the interventricular septum was thickened in all mutant hearts (Fig. 4A). Gross observation and serial sections of E18.5 and postnatal day 1 (P1) pups indicated proper partitioning of the great vessels and closure of the ductus arteriosis (Supplemen- tary Fig. 1; data not shown). Histological analysis showed no evidence of fat deposition or fibrosis in the myocardium of HDAC2 mutant neonates (data not shown).

ECGs on mice at P1 showed an average decreased heart rate of $152 \pm 14$ beats per minute (bpm) compared with $235 \pm 20 \mathrm{bpm}$ in wild-type littermates (Fig. 4B). While all $H D A C 2^{-/-}$mice were bradycardic, we never observed arrhythmias or defects in atrioventricular conduction in the mutant animals. In all ECGs and rhythm strips taken from knockout mice, normal p-wave morphology was observed (data not shown). Moreover, all p-waves were upright in lead II, suggesting that the location of the pacemaker lies high in the right atrium in or near the sinus node. These observations suggest that these mice are most likely in normal sinus rhythm.

\section{Increased cardiac apoptosis and proliferation in HDAC2 mutant mice}

Given the diminished right ventricular lumen and increased interventricular wall thickness of $\mathrm{HDAC2}^{-/-}$ 
Montgomery et al.

A

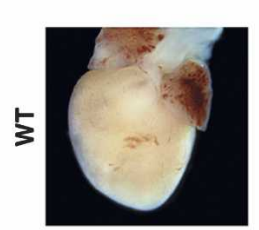

Level 1
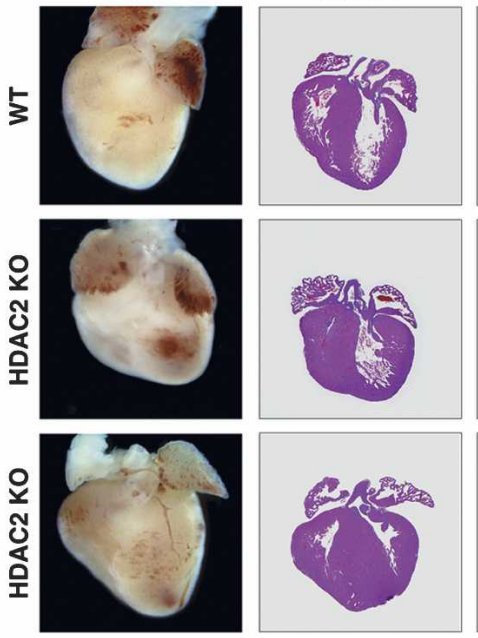

B

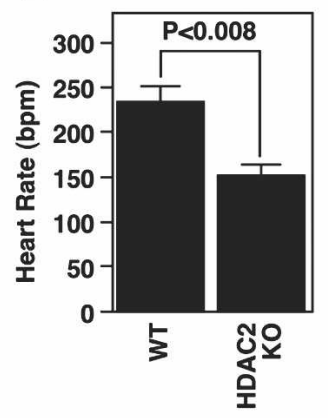

D

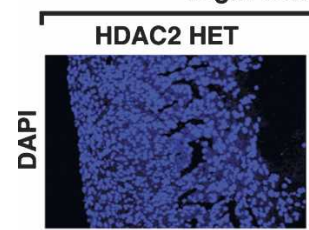

Right Ventricle (20x) HDAC2 KO
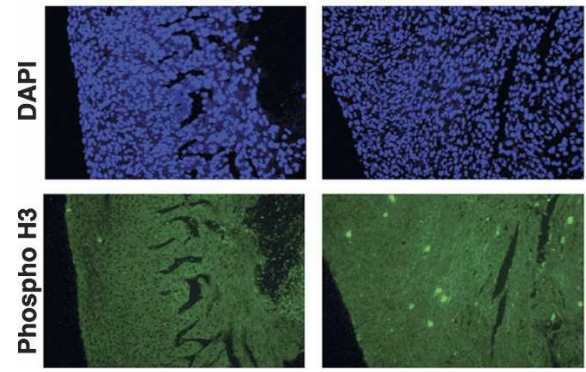

Level 2
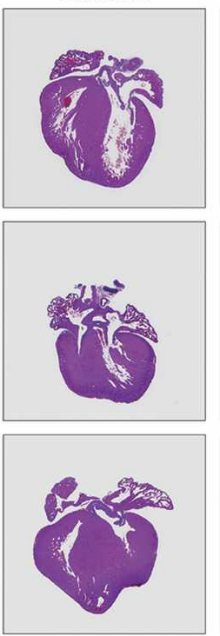

Level 3
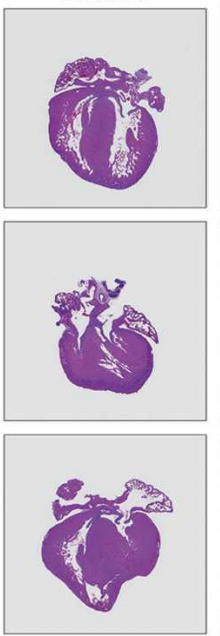

C

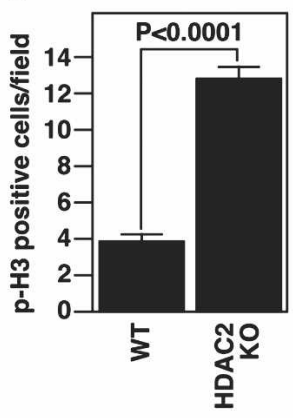

Level 4
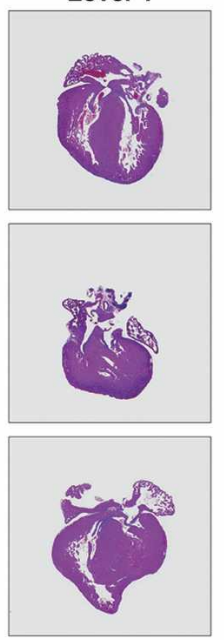

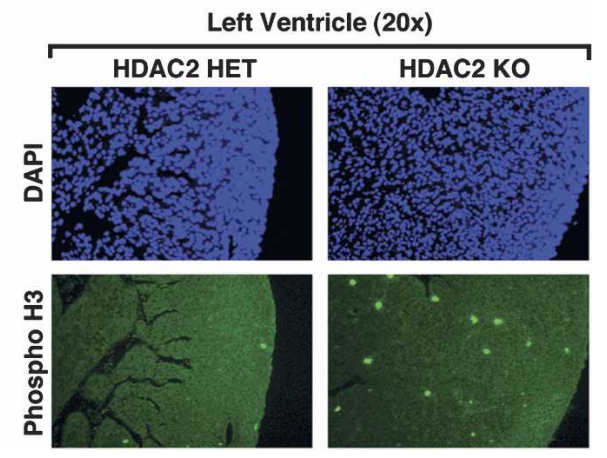

Figure 4. Cardiac defects in HDAC2 ${ }^{-/-}$neonates. (A) Structural abnormalities in the hearts of HDAC2-null neonates. Hearts from mice of the indicated genotypes at P1 are shown at the left. H\&E-stained sections at four successive levels of wild-type and HDAC2null hearts are shown. The myocardium of HDAC2-null animals displays a thicker septum and diminished or dislocated right ventricular lumen. (B) Heart rates of HDAC2-null neonates. EKGs show reduced heart rate in the HDAC2-null mice. (C) Increased proliferation of cardiomyocytes in HDAC2-null mice. Immunohistochemistry for phospho-histone H3 was performed on heart sections from P1 mice. Quantification of phospho-histone H3-positive cells was performed on six sections from three individual hearts and averaged. $(D)$ Immunohistochemistry of heterozygous and HDAC2-null hearts at 20× magnification. DAPI (blue) and phosphohistone H3 (green) staining show increased proliferation in both the right and left ventricles.

mice, we performed phospho-histone H3 staining to distinguish between cardiomyocyte hyperplasia and hypertrophy. Mutant hearts displayed a threefold increase in phospho-histone $\mathrm{H} 3$ staining compared with wild-type hearts at P1 $(P<0.0001)$ (Fig. 4C). Enhanced phosphohistone $\mathrm{H} 3$ staining was observed throughout the right ventricle, as well as the interventricular septum and left ventricular free wall (Fig. 4D). Increased proliferative activity was not observed in any other tissue examined, including brain, lung, liver, thymus, or skeletal muscle (data not shown).

Terminal deoxynucleotidyl transferase biotin-dUTP 
nick end labeling (TUNEL) analysis revealed increased apoptosis in both right and left ventricular walls as well as the interventricular septum (data not shown), which was most pronounced at the base of the interventricular septum of mutant hearts at P1 (Supplementary Fig. 2). TUNEL analysis on extra-cardiac tissue showed no differences between wild-type and mutant animals (data not shown).

\section{Cardiac deletion of HDAC2}

To determine if the neonatal lethality of HDAC2-null mice was due solely to a cardiomyocyte-autonomous function of HDAC2, we deleted HDAC2 specifically in the heart by breeding homozygous $H D A C 2^{\text {IoxP/loxP }}$ mice to the $\alpha M H C$-Cre transgenic line. In contrast to the global deletion of HDAC2, HDAC2 ${ }^{\text {loxP/loxP }}$; $\alpha M H C$-Cre mice were viable to adulthood and showed no gross cardiac abnormalities (data not shown). HDAC2 immunostaining of cardiomyocytes from $H D A C 2^{\operatorname{loxP} / \text { /oxP }}$; $\alpha M H C$-Cre mice confirmed the absence of HDAC2 protein in cardiomyocytes (Supplementary Fig. 3).

We further crossed mice bearing the conditional $H D A C 2$ allele to a variety of Cre lines to delete the gene in the neural crest, skeletal muscle, central nervous system, endothelium, smooth muscle, and secondary heart field (Supplementary Table 1). No Cre line homozygous for $H D A C 2^{\operatorname{loxP} / / \operatorname{lox}}$ was able to phenocopy the global deletion of $H D A C 2$, suggesting that the lethal cardiac abnormalities of $H D A C 2^{-/-}$mice reflected functions of HDAC2 in multiple cell types within the heart.

\section{Cardiac deletion of HDAC1 and HDAC2 causes heart failure and aberrant gene expression}

To test for possible functional redundancy between HDAC1 and HDAC2 in cardiomyocytes, we generated cardiac-specific deletions of both genes using $\alpha M H C$ Cre. A single copy of either allele was sufficient for viability, whereas double deletion of HDAC1 and HDAC2 by $\alpha M H C$-Cre (designated dCKO for double conditional cardiac knockout) resulted in postnatal lethality by $14 \mathrm{~d}$ (Fig. 5A). Hearts from dCKO mice appeared grossly normal at P8, but displayed cardiac arrhythmias by P10 and severe right and left ventricular dilatation by P11 (Fig. $5 B, C)$. TUNEL staining revealed an approximately threefold increase in apoptosis in the hearts of dCKO mice compared with wild-type or $H D A C 1^{\operatorname{lox} P / / 0 x P}$; $H D A C 2^{\operatorname{lox} P /+}$; $\alpha M H C$-Cre littermates $(P<0.0001)$ (Fig. 5D,E).

We examined multiple markers of the cardiac stress response in $\mathrm{dCKO}$ mice and found the cardiac stress markers atrial natriuretic factor (ANF), brain natriuretic peptide (BNP), $\beta$-myosin heavy chain ( $\beta M H C)$, $\alpha$-skeletal actin, and MLC-2a to be dramatically up-regulated at P11, consistent with the heart failure phenotype of these mice (Fig. 5F).

To further understand the primary cause of the observed heart failure phenotype, we performed expression profiling of dCKO hearts at $\mathrm{P} 8$. At this time point, hearts were morphologically normal (Fig. 5B) and cardiac stress markers were either unchanged or only mildly induced (data not shown). Surprisingly only a small portion of the transcriptome was dysregulated with $0.94 \%$ (162 genes) of unique and annotated transcripts up-regulated and $0.58 \%$ (100 genes) down-regulated when a cutoff of twofold change was applied (Supplementary Tables 2, 3).

To analyze if certain pathways or biological processes were especially sensitive to the loss of HDAC1 and HDAC2 in the heart, a gene ontology analysis (Thomas et al. 2003) was performed on the dysregulated transcripts. This analysis indicated that the most significantly enriched up-regulated genes participate in cell structure and motility (Fig. 6A). Up-regulated genes falling in this group were further analyzed for their annotated biological function, showing that the majority of the up-regulated genes encode either cytoskeletal proteins or ion channels (Fig. 6B).

\section{Up-regulation of calcium channel genes resulting from cardiac deletion of HDAC1 and 2}

Microarray analysis of hearts from dCKO mice at P8 revealed inappropriate up-regulation of specific L-type and T-type calcium channel subunit genes. $\mathrm{Ca}_{\mathrm{v}} 3.2$, a T-type channel encoded by $C A C N A 1 H$, is normally expressed in embryonic hearts and is down-regulated after birth (Yasui et al. 2005). Microarray analysis revealed a 3.5fold increase in expression of $\mathrm{Ca}_{\mathrm{v}} 3.2$, which was confirmed by real-time PCR at P8, and even greater up-regulation at P11 (Fig. 6C). Induced expression of $\mathrm{Ca}_{\mathrm{V}} 3.2$ in dCKO hearts was also detected by Western blot analysis (Fig. 6D). The L-type calcium channel subunit $\alpha 2 \delta 2$, encoded by CACNA2D2, was also up-regulated 8.6-fold at $\mathrm{P} 8$ and to even higher levels at P11 in dCKO mice (Fig. 6C). These genes appeared to be specific targets for repression by $\mathrm{HDAC} 1$ and $\mathrm{HDAC} 2$, as there were no significant changes in expression of other regulators of calcium handling, including the sarco(endo/plasmic reticulum $\mathrm{Ca}^{2+}$-ATPase, SERCA2; the ryanodine receptor 2, RyR2; or the $\mathrm{Na}^{+} / \mathrm{Ca}^{2+}$ exchanger, NCX1 (Fig. 6C; data not shown). In addition, analysis of transcript levels for CACNA1H and CACNA2D2 in HDAC1 $1^{\text {1oxP/loxP }}$; $\alpha M H C$ Cre and $H D A C 2^{\operatorname{loxP} / \text { loxP }} ; \alpha M H C$-Cre mice showed no significant increase in expression (Fig. 6C), suggesting HDAC1 and HDAC2 redundantly regulate the expression of L-type and T-type calcium channel subunits during postnatal development.

$\mathrm{Ca}_{\mathrm{v}} 3.2$ has been shown to be repressed by neuron-restrictive silencing factor (NRSF), and up-regulation of $\mathrm{Ca}_{\mathrm{v}} 3.2$ has been shown to render the heart susceptible to cardiac arrhythmias and sudden death (Kuwahara et al. 2003). To investigate whether HDAC1 and HDAC2 also regulate $\alpha 2 \delta 2$ in an NRSF-dependent manner, we performed chromatin immunoprecipitation (ChIP) on neonatal rat ventricular myocytes. Immunoprecipitation of HDAC1, HDAC2, or NRSF pulled down the neuron-restrictive silencing element (NRSE)-containing region within intron 1 of CACNA2D2, whereas anti-HA as a negative control did not recognize this regulatory DNA sequence. These findings suggest that a repressive complex containing NRSF/HDAC1/HDAC2 normally re- 
Montgomery et al.

Figure 5. Cardiac defects resulting from cardiac deletion of HDAC1 and HDAC2. (A) Kaplan-Meier survival curves for cardiac deletion of HDAC1 and HDAC2 by $\alpha M H C$-Cre. Note that one copy of HDAC2 is sufficient for $100 \%$ viability. (B) H\&Estained sections of wild-type and dCKO mice at P8, P11, and P13. Deletion of both HDAC1 and HDAC2 results in severe dilated cardiomyopathy by P11. (C) ECGs performed on wild-type and dCKO mice at P10. Deletion of HDAC1 and HDAC2 in cardiomyocytes leads to cardiac arrhythmia by P10. (D) Apoptosis in dCKO mice. TUNEL staining of wild-type and dCKO ventricular sections showed enhanced apoptosis in dCKO mice at P10. (E) Quantification of apoptosis in dCKO hearts. TUNEL-positive cells were quantified from six individually stained sections at $40 \times$ and averaged; $P<0.0001$. (F) Expression of cardiac stress markers in dCKO mice at P11. mRNA transcript levels were detected by real-time RT-PCR and normalized to 18S RNA.
A
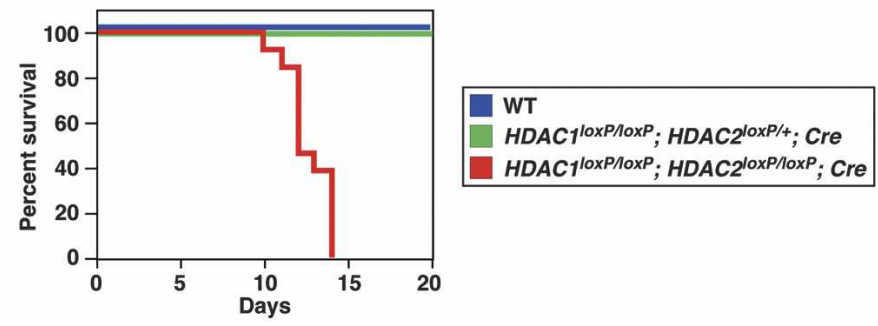

\section{B}
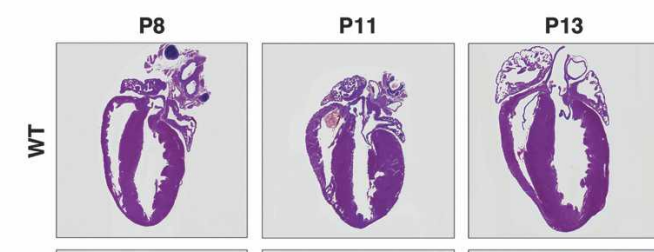

C
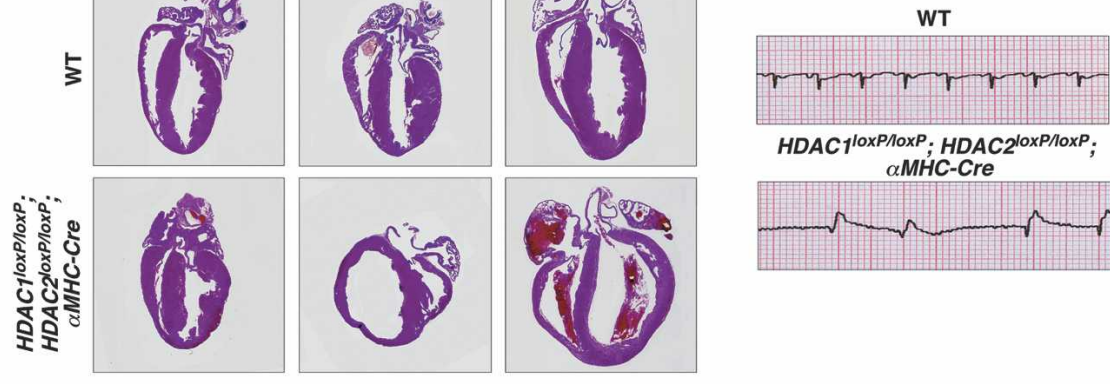

HDAC1 $1^{\text {loxPRloxP; HDAC }}$ loxP/loxP;

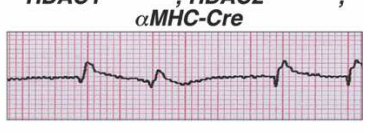

D
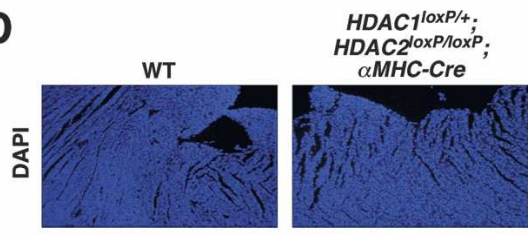

HDAC1 10xP/loxp; HDAC2 $2^{\text {IoxP/oxP }}$
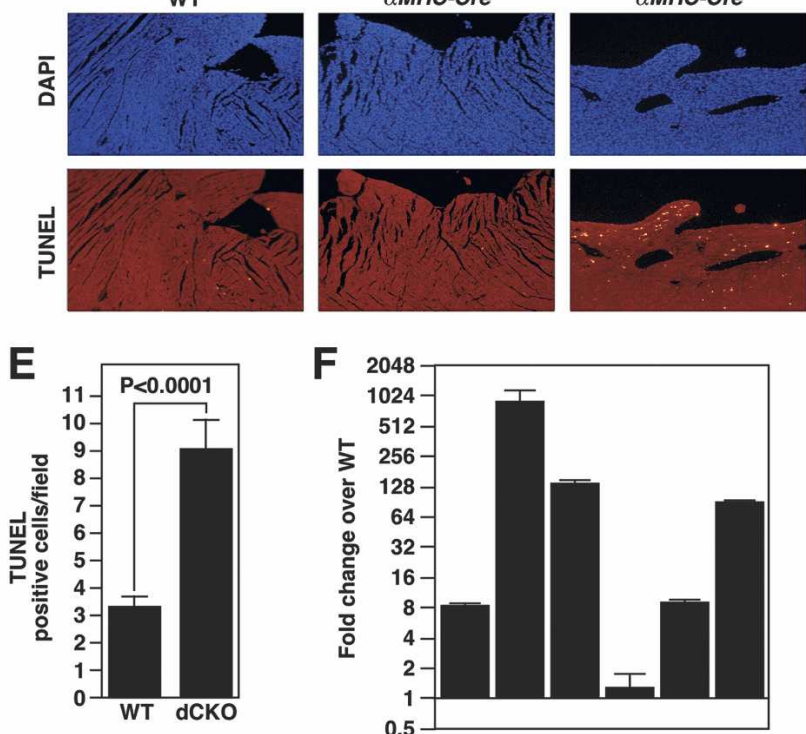
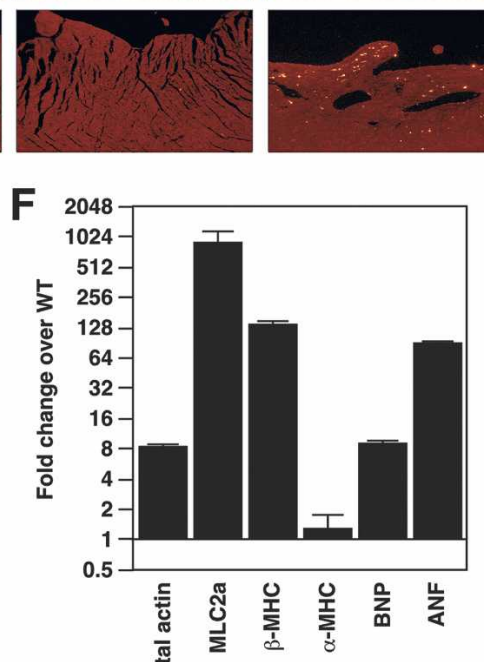

presses ventricular expression of $\alpha 2 \delta 2$ under basal conditions (Fig. 6E).

Up-regulation of skeletal muscle contractile protein genes resulting from cardiac deletion of HDAC1 and HDAC2

Cardiac stress is often accompanied by the up-regulation of skeletal muscle contractile protein genes in the heart, which alters calcium handling and increases cardiac contractility. Indicative of heart failure, the expression of $\alpha$-skeletal actin (Acta1), myosin light chain 1F (MLC1f), and skeletal isoform of troponin $\mathrm{T}$ (Tnnt3) was up-regulated in dCKO mice (Figs. 5F, 6F). Notably, the ventricular isoform of myosin light chain, $M L C 1 \mathrm{~V}$, was not induced, arguing against a global dysregulation of contractile proteins.

Strikingly, troponin I (TnI), an inhibitory subunit of the heterotrimeric troponin complex that allows for the binding of actin to myosin in response to influxes of $\mathrm{Ca}^{2+}$, resulting in sarcomeric activation and contraction (Parmacek and Solaro 2004), was the most dramatically 
A

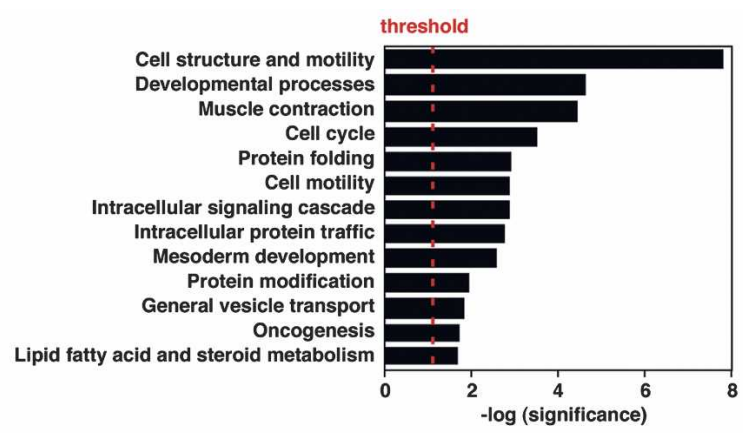

C

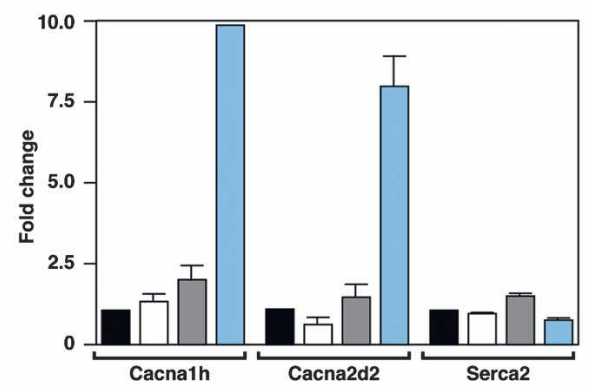

F

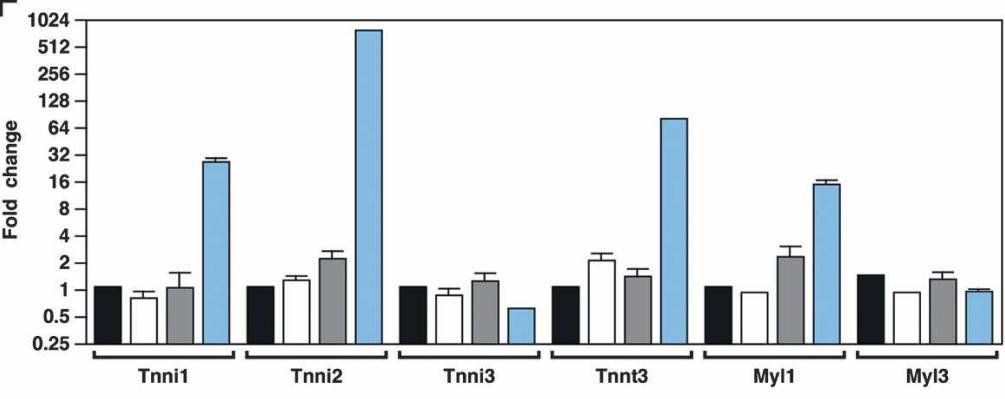

G

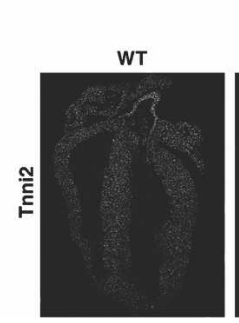

HDAC1loxPAoxP. HDAC210xP/oxp

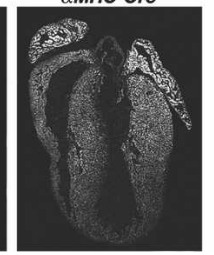

B

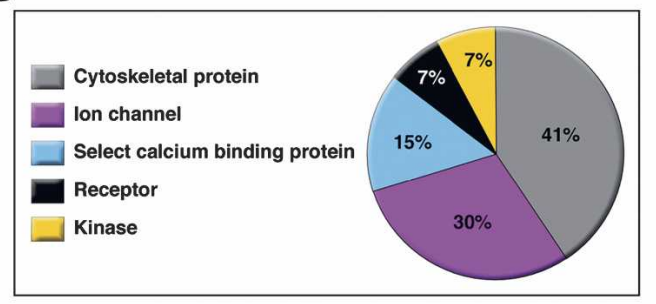

D

E

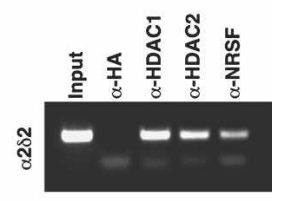

wT

HDAC1 10xPRoxp; $\alpha$ MHC-Cre

HDAC2 $20 \times P$ NoxP; $\alpha \mathrm{MHC}-\mathrm{Cre}$

dCKO

Figure 6. Aberrant cardiac gene expression resulting from cardiac deletion of HDAC1 and HDAC2. $(A)$ Gene ontology analysis was performed with PANTHER. Significantly $(P<0.05)$ enriched biological processes are shown. Plotted is the $-\log (P$ value $)$ with the threshold set to $1.3[\log (0.05)]$. (B) Molecular functions were assigned to the genes that fall in the most significantly enriched process, "cell structure and motility" $(P<0.00000005) .(C)$ Calcium channel subunit dysregulation in the hearts of dCKO mice. Real-time RT-PCR analysis of transcript levels from wild-type, cardiac-specific HDAC1 KO, cardiac-specific HDAC2 KO, and dCKO hearts at P11. L-type and T-type calcium subunits were dysregulated in the dCKO hearts. Error bars indicate standard deviation. $(D) \mathrm{Ca}_{\mathrm{V}} 3.2$ expression in dCKO hearts. Western blot analysis was performed on hearts from P11 wild-type (lane 1) and dCKO (lanes 2,3) mice. dCKO mice show increased expression of $\mathrm{Ca}_{\mathrm{V}} 3.2$. Tubulin was detected as a loading control. (E) ChIP assays were performed from neonatal rat ventricular myocytes. Chromatin was immunoprecipitated with antibodies against HA as a negative control, HDACl, HDAC2, or NRSF. Primers were designed around the NRSE within intron 1 of CACNA2D2, and precipitated DNA was analyzed by PCR. PCR was also performed from a nonimmunoprecipitated sample as an input control. (F) Specific dysregulation of skeletal myofibrillar proteins in the hearts of dCKO mice. Real-time RT-PCR analysis of transcript levels from wild-type, cardiac-specific HDAC1 KO, cardiac-specific HDAC2 KO, and dCKO hearts at P11. Error bars indicate standard deviation. $(G)$ RNA in situ hybridization of Tnni2 transcripts on wild-type and dCKO hearts. $(H)$ Troponin isoform expression in dCKO heats. Western blot analysis was performed on hearts from P11 wild-type (lane 1) and dCKO (lanes 2,3) mice. dCKO mice show heterogeneity in troponin isoform expression. Lane 4 is wild-type skeletal muscle as a control for Tnni2. (I) ChIP assays were performed on the IRE of Tnni2 in neonatal rat ventricular myocytes. Chromatin was immunoprecipitated with antibodies against HA, HDAC1, HDAC2, and NRSF. Precipitated DNA was analyzed by PCR using primers flanking the IRE. HDAC1 and HDAC2, but not NRSF, mediate repression of Tnni2 at basal levels. PCR was performed prior to immunoprecipitation as an input control. 
up-regulated gene in the mutant heart. There are three mammalian isoforms of TnI: a cardiac TnI (Tnni3), and a slow and fast skeletal TnI (Tnni1 and Tnni2, respectively). Both skeletal muscle isoforms were induced in the dCKO mice at P11. Remarkably, the fast skeletal isoform, Tnni2, was induced $>500$-fold (Fig. 6 F). In addition, the levels of the cardiac-specific isoform were unchanged, if not moderately down. Analysis of transcript levels in hearts with cardiac-specific deletions of either HDAC1 or HDAC2 showed no significant changes in skeletal muscle myofibril proteins (Fig. 6F), further suggesting $\mathrm{HDAC} 1$ and $\mathrm{HDAC} 2$ as redundant regulators of myofibril gene expression.

RNA in situ hybridization on P8 hearts confirmed the induced expression of Tnni2, not only in the ventricles but in the atria of dCKO mice as well (Fig. 6G). Cardiac expression of all three isoforms of TnI was also detected by Western blot analysis using a pan-troponin antibody (Fig. 6H). The skeletal muscle-specific isoforms of TnI are not typically induced during cardiac hypertrophy or failure (Sasse et al. 1993; Bodor et al. 1997), suggesting the induction of Tnni1 and Tnni2 is not compensatory but represents a primary defect contributing to the cardiac arrhythmia leading to sudden death.

ChIP assays on neonatal rat cardiomyocytes showed that HDAC1 and HDAC2 were localized to the homologous internal regulatory element (IRE) (Fig. 6I), which has been shown to direct skeletal muscle-specific expression of Tnni2 (Lin et al. 1991). Although there is an NRSFbinding site within the IRE of the Tnni2 gene, NRSF was not detected on this element in neonatal rat cardiomyocytes (Fig. 6I), suggesting that HDAC1 and HDAC2 repress Tnni2 through an NRSF-independent mechanism.

\section{Hypertrophic response to adrenergic stimulation} and aortic constriction

Based on the ability of HDAC inhibitors to blunt hypertrophic growth of cardiomyocytes in response to stress (Antos et al. 2003; Kee et al. 2006; Kong et al. 2006), we tested whether cardiac deletion of HDAC1 or HDAC2 diminished the hypertrophic growth response to chronic infusion of isoproterenol, a $\beta$-adrenergic agonist that induces cardiac hypertrophy, or thoracic aortic constriction (TAC), which induces cardiac hypertrophy through increased afterload on the heart. As shown in Figure 7, $H D A C 1^{\operatorname{lox} P / \operatorname{lox} P} ; \alpha M H C-C r e$ mice and $H D A C 2^{\operatorname{lox} P / / \operatorname{lox} P}$; $\alpha M H C$-Cre mice displayed a hypertrophic response to isoproterenol that was comparable to that of wild-type littermates. Similarly, HDAC1 $1^{\text {loxP/loxP }} ; \alpha M H C$-Cre mice showed no statistical difference in heart weight/body weight (HW/BW) or heart weight/tibia length (HW/TL) ratios compared with HDAC1 $1^{\text {loxP/loxP }}$ littermates not expressing Cre recombinase following TAC (Fig. 7B). Histological analyses were also indistinguishable between $H D A C 1^{\text {loxP/loxP }}$; $\alpha M H C$-Cre mice and littermate controls (Fig. 7C).

\section{Discussion}

Class I HDACs are expressed ubiquitously and are thought to function as global repressors of transcription.
A

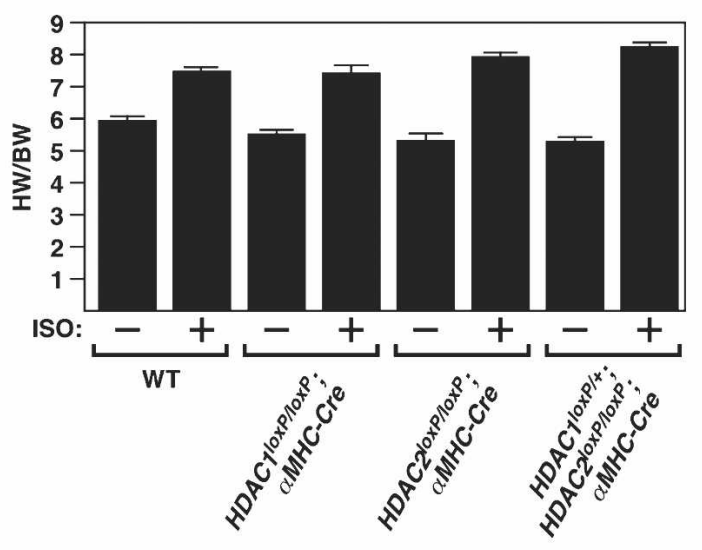

B
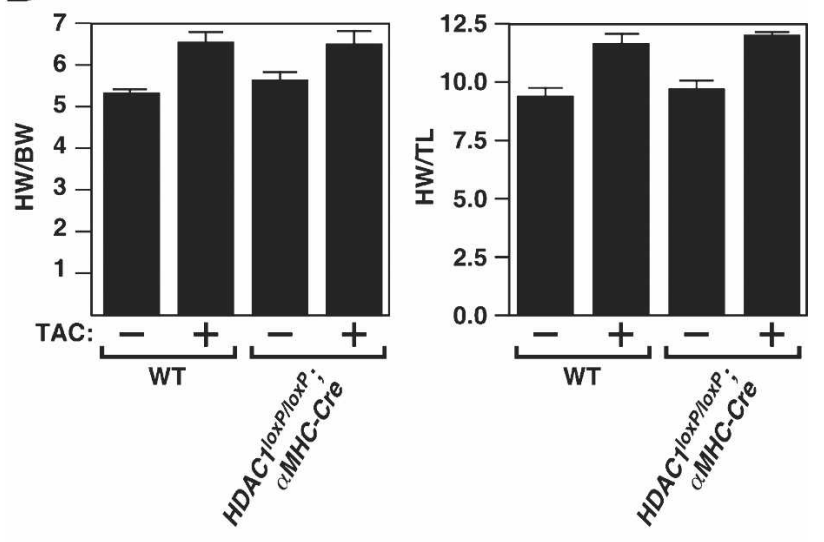

C

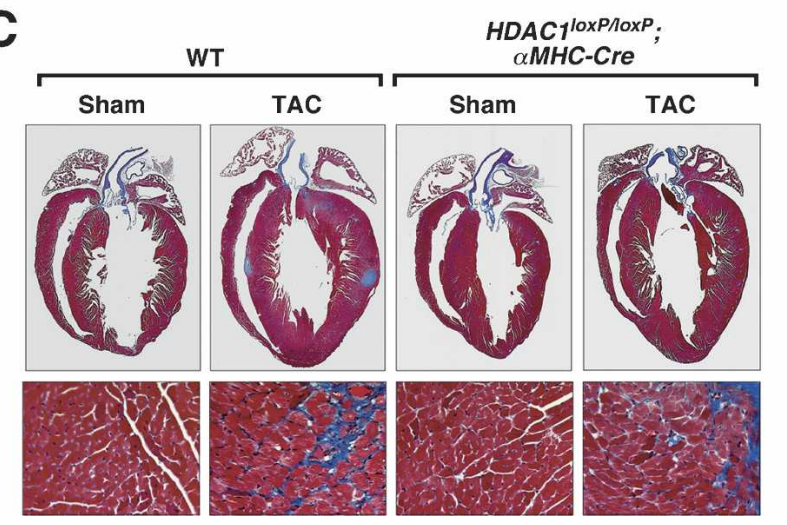

Figure 7. Stress-dependent cardiac hypertrophy in mice lacking cardiac expression of HDAC1 and HDAC2. (A) Wild-type, HDAC1 $1^{\text {loxP/loxP }} ; \alpha M H C-C r e, H D A C 2^{\text {loxP/loxP }} ; \alpha M H C-C r e$, and $H D A C 1^{10 x P /+} ; H D A C 2^{10 x P / l o x P} ; \alpha M H C$-Cre mice at $8 \mathrm{wk}$ of age were subjected to isoproterenol or saline infusion. Mice were sacrificed after $7 \mathrm{~d}$, and cardiac hypertrophy was evaluated by heart weight/body weight ratios. (B) Wild-type and HDAC $1^{\text {loxP/loxP }}$; $\alpha M H C$-Cre mice were subjected to TAC or sham operation. Heart weight/body weight ratios and heart weight/tibia length ratios were determined after $21 \mathrm{~d}$. (C) Histological sections of representative hearts from $B$ are shown stained with Masson Trichome. Bottom panels are 40× magnifications of the above hearts to show fibrosis.

The results of this study provide the first glimpse of the functions of $\mathrm{HDAC} 1$ and $\mathrm{HDAC} 2$ in postnatal tissues in 
vivo and reveal specialized and redundant roles of these HDACs in the control of cardiac growth and function.

\section{Roles of HDAC1 and HDAC2 in cardiac growth and function}

Mice homozygous for an HDAC1-null allele die at gastrulation from a block to cell proliferation due, at least in part, to up-regulation of the cell cycle inhibitor p21 (Lagger et al. 2002). Thus, the potential functions of HDACl at later stages of embryogenesis or after birth have remained enigmatic. Our results show that HDAC1 can be deleted in a wide range of tissues, including cardiac, skeletal and smooth muscle, neural crest, endothelial cells, and the central nervous system, without affecting viability. The essential early role of HDACl is, therefore, likely to reflect a specific requirement at gastrulation rather than a general requirement in cell proliferation per se.

Our results show that HDAC2 is essential for normal growth and morphogenesis of the heart. Global deletion of HDAC2 results in a highly specific cardiac phenotype associated with uncontrolled proliferation of ventricular cardiomyocytes, which leads to obliteration of the right ventricular chamber, causing perinatal lethality with complete penetrance. These defects appeared to be superficially similar to many known congenital heart abnormalities, including hypoplastic right heart syndrome, right ventricular dysplasia, and transposition of the great arteries. However, histological analysis at the cellular level showed no infiltration of fat and no signs of fibrosis in HDAC2 mutant neonates, which typically accompany RV dysplasia. In addition, there were no signs of pulmonary valve atresia, a common defect in hypoplastic right heart syndrome. The finding that conditional deletion of HDAC2 in cardiomyocytes, smooth muscle, endothelial cells, and neural crest does not phenocopy the cardiac defects observed in HDAC2-null mice suggests that HDAC2 may be required in multiple cell types for normal heart development.

The neonatal lethal phenotype we observed for HDAC2null mice contrasts with that of another recent study in which mice homozygous for a gene trap insertion mutation in $H D A C 2$, purported to create a null allele, were viable (Trivedi et al. 2007). We cannot explain the basis for these differing phenotypes, but there are several possibilities. LacZ insertion lines frequently display variable phenotypes due to alternative splicing within the mutant locus (Voss et al. 1998). Thus, it is conceivable that the mutation described by Trivedi et al. (2007) is a hypomorphic allele, rather than a true null, and generates sufficient levels of HDAC2 for viability of a subset of mutant animals. The mutation we introduced into the HDAC2 locus deleted many key residues required for deacetylase activity (Finnin et al. 1999), whereas that gene trap insertion ablates the $\mathrm{C}$ terminus of the protein that, to date, has unknown function (Gregoretti et al. 2004). Different genetic backgrounds of the mice tested in the two studies could also contribute to the differing phenotypes. The mice in our study were from a mixed 129/C57BL/6/CD1 background, while the background of the mice generated by Trivedi et al. (2007) was not specified.
Our findings also differ from those of Trivedi et al. (2007) with respect to the role of HDAC2 in mediating stressdependent cardiac growth. Diverse stresses, including neurohormonal signaling and pressure overload, promote pathological cardiac hypertrophy, which often culminates in heart failure and lethal cardiac arrhythmias. HDAC inhibitors have shown efficacy both in vitro and in vivo in blunting the hypertrophic response (Antos et al. 2003; Kee et al. 2006; Kong et al. 2006). Our results demonstrate that cardiac deletion of either HDAC1 or HDAC2 is not sufficient to block hypertrophy in response to chronic administration of isoproterenol or aortic constriction. In contrast, Trivedi et al. (2007) reported that the lacZ insertional mutation in HDAC2 prevented cardiac hypertrophy in response to these stimuli.

The finding that cardiac deletion of either HDACl or HDAC2 has no apparent effect on cardiac development or function whereas deletion of both genes in the heart results in lethal cardiac abnormalities strongly suggests that these HDACs act in a redundant manner to regulate cardiac gene expression. Based on the high sequence homology between HDAC1 and HDAC2 and the cardiac phenotype resulting from the combined deletion of these genes, it is likely that these genes play redundant roles in many tissues. Double tissue-specific gene deletions to address this issue are underway.

\section{Abnormalities in gene expression resulting from HDAC1 and HDAC2 deletion}

Deletion of two global transcriptional repressors such as HDAC1 and HDAC2 might be expected to result in rampant ectopic gene activation. Instead, the consequences of HDAC1 and HDAC2 loss of function in the heart were remarkably specific and resulted in selective induction of genes involved in calcium influx, calcium handling, and contraction.

In cardiomyocytes, the thin filaments, together with the myosin-containing thick filaments, regulate $\mathrm{Ca}^{2+}$ sensitivity, contractility, and relaxation. The thin filament, which is composed of actin, tropomyosin, and the heterotrimeric troponin complex, responds to intracellular $\mathrm{Ca}^{2+}$ fluxes from $\mathrm{Ca}^{2+}$ channels to control cardiac contractility (Kobayashi and Solaro 2005). The troponin complex is comprised of a $\mathrm{Ca}^{2+}$-binding troponin $\mathrm{C}$ $(\mathrm{TnC})$, a tropomyosin-binding troponin $\mathrm{T}(\mathrm{TnT})$, and an inhibitory subunit (TnI) that blocks actin-myosin binding at low $\mathrm{Ca}^{2+}$ levels (Parmacek and Solaro 2004). The absence of HDAC1 and HDAC2 results in the up-regulation of $\mathrm{T}$ - and L-type $\mathrm{Ca}^{2+}$ channels as well as robust expression of the skeletal muscle-specific isoforms of $\mathrm{TnI}$ (ssTnI and fsTnI), which exhibit varying sensitivities to intracellular $\mathrm{Ca}^{2+}$ and are regulated independently through phosphorylation (Parmacek and Solaro 2004).

Expression of the T-type calcium channel $\mathrm{Ca}_{\mathrm{v}} 3.2$ is regulated by the transcriptional repressor NRSF (Kuwahara et al. 2003), which recruits class I HDACs (Roopra et al. 2000). By ChIP assays, we identified HDAC1, HDAC2, and NRSF on the NRSE in intron 1 of CACNA2D2 in neonatal rat cardiac myocytes, suggesting class I HDACs actively 
repress the expression of $C A C N A 2 D 2$ during normal cardiac growth. HDAC1 and HDAC2 were also localized to the IRE of Tnni2; however, this appears to be in an NRSFindependent manner. We postulate that the increased expression of $\mathrm{Ca}^{2+}$ channel subunits results in a pathological influx of $\mathrm{Ca}^{2+}$ into cardiomyocytes that, coupled with a heterogeneous population of $\mathrm{Ca}^{2+}$-sensitive TnI, results in a dysregulation of sarcomeric activation, contraction, and relaxation, resulting in cardiac arrhythmia and sudden death.

\section{Implications for human disease}

HDACs have been implicated in a wide range of cellular processes and disease states based on the ability of HDAC inhibitors to ameliorate various disease pathologies (Bolden et al. 2006; Minucci and Pelicci 2006). However, an important issue that has been difficult to resolve is whether the effects of HDAC inhibitors in vivo reflect the combined inhibition of multiple HDACs or specific functions of individual HDACs in different tissues. The conditional alleles of class I HDACs will allow the detailed analysis of the functions of these chromatin remodeling enzymes in diverse tissues during development and in the settings of disease. Moreover, by testing the responses to HDAC inhibitors of cells isolated from these mutant mice, it should be possible to identify the specific HDAC isoforms that mediate the actions of HDAC inhibitors in vivo. Finally, it should be pointed out that the cardiac abnormalities resulting from genetic deletion of HDAC1 and HDAC2 reflect the consequences of the complete absence of these HDACs throughout development and should not be interpreted as a contraindication for the use of HDAC inhibitors in the treatment of adult heart disease, especially since pharmacological inhibition is not as complete as genetic ablation.

\section{Materials and methods}

Generation of HDAC1 and HDAC2 mutant mice

Both HDAC1 and HDAC2 targeting vectors were constructed using the pGKNEO-F2L2DTA vector, which contains a neomycin resistance gene, flanked by FRT and loxP sites, and a diphtheria toxin gene cassette. The $5^{\prime}$ arm, $\mathrm{KO}$ arm, and $3^{\prime}$ arm of the targeting construct for HDAC1 were generated by high-fidelity PCR amplification (Roche Expand High-Fidelity Long Template) of $129 \mathrm{SvEv}$ genomic DNA and correlate to a $2-\mathrm{kb}$ fragment in intron 4, a 2-kb fragment harboring exons $5-7$, and a $4-k b$ fragment in intron 7, respectively. For the targeting construct of $H D A C 2$, a 4 -kb fragment in intron 1 , a 4-kb fragment harboring exons 2-4, and a 2-kb fragment in intron 4 were generated correlating to the $5^{\prime}$ arm, $\mathrm{KO}$ arm, and 3' arm, respectively. Both targeting vectors were linearized with PvuI and electroporated into 129SvEv-derived ES cells. One-thousand (for HDAC1) and 400 (for HDAC2) ES cell clones were isolated and analyzed for homologous recombination by Southern blotting. 5' $10 x P$ incorporation for HDAC1 was confirmed using a $5^{\prime}$ probe following digestion with EcoRI. HDAC2 long arm and $5^{\prime}$ loxP recombination were confirmed using a $5^{\prime}$ probe following digestion with XbaI. Short arm and 3' lox $P$ confirmation were performed using a $3^{\prime}$ probe after EcoRI or SacI digestion for $H D A C 1$ and $H D A C 2$, respectively. Three clones with a properly targeted HDAC1 or HDAC2 allele were injected into 3.5-d C57BL/6 blastocysts, and the resulting chi- meras were crossed to C57BL/6 females to achieve germline transmission of the targeted allele.

\section{Histology, immunohistochemistry, and RNA} in situ hybridization

Tissues were fixed in $4 \%$ paraformaldehyde, embedded in paraffin, and sectioned at 5 - $\mu \mathrm{m}$ intervals. Sections were stained with hematoxylin and eosin using standard procedures (Shelton et al. 2000). TUNEL assay was performed according to standard protocol (Roche), and immunohistochemistry using an anti-rabbit phospho-histone $\mathrm{H} 3$ antibody was performed as described (Xin et al. 2006). ${ }^{35}$ S-labeled RNA probes were generated using Maxiscript kit (Amersham).

\section{RT-PCR, microarray, and gene ontology analyses}

Total RNA was purified from tissues using TRIzol reagent according to the manufacturer's instructions. For RT-PCR, total RNA was used as a template for RT using random hexamer primers. Primer sequences are available on request. Quantitative real-time PCR was performed using TaqMan probes purchased from ABI. For microarray, RNA was extracted from either three wild-type or dCKO hearts and subsequently pooled prior to analysis. All heart RNA was from ventricle tissue only. Microarray analysis was performed by the University of Texas Southwestern Microarray Core Facility using the Mouse Genome 430 2.0 Array (Affymetrix) as described (Davis et al. 2006). Gene Ontology analysis with PANTHER (Thomas et al. 2003) was performed essentially as described (Matsuoka et al. 2007). Briefly, 1586 probe spots were flagged as significantly up-regulated by the Affymetrix analysis software GeneChip. The corresponding Entrez Gene IDs (Maglott et al. 2007) were uploaded to PANTHER, and 1176 unique and annotated genes could be identified from this data set that then could be assigned to 197 different biological processes. This distribution was compared with a reference list (NCBI: Mus musculus genes, version 1-162007) (Maglott et al. 2007), and significant enrichment of the experimental data set in a given process was calculated using binomial testing with Bonferroni correction for multiple testing as described (Thomas et al. 2006). Only processes with a significant $(P<0.05)$ enrichment were used for further analysis. The process with the highest significance score was then analyzed for molecular function using PANTHER. Only families with more than one gene in a given family were considered.

The percentage of gene changes was calculated by uploading the Entrez Gene IDs of the Mouse Genome 430 2.0 Affymetrix annotation file to PANTHER, leading to the identification of 17,201 unique and annotated transcripts. These were then compared with the statistically significantly dysregulated unique and annotated transcripts (ESTs excluded) as identified by GeneChip using a cutoff at twofold change. Similar results were obtained when nonannotated transcripts were included in the analysis (data not shown).

\section{Indirect immunofluorescence}

Hearts were extracted from 2- to 3-d-old wild-type and dCKO mice, minced in DMEM (Cellgro), washed in PBS, and digested with collagenase type II $(0.2 \% \mathrm{w} / \mathrm{v}$; Worthington Biochemical $)$ and glucose $(0.1 \% \mathrm{w} / \mathrm{v}$; Sigma). Cells were resuspended in DMEM containing fetal bovine serum (FBS; 10\%), L-glutamine (2 $\mathrm{mM})$, and penicillin-streptomycin. Cells were preplated twice for $2 \mathrm{~h}$ each to separate adherent fibroblasts from cardiomyocytes. Cardiomyocytes were plated on gelatin-coated glass coverslips in six-well dishes $\left(4 \times 10^{5}\right.$ cell per well). After $24 \mathrm{~h}$, cells were fixed with ice-cold methanol, permeabilized, and blocked with PBS containing Nonidet P-40 (PBS-N) $(0.1 \%)$ and 
bovine serum albumin (BSA; $3 \%$ ) and incubated in the same solution for primary antibodies for HDAC1 (rabbit polyclonal, 1:2000 dilution; Abcam), HDAC2 (rabbit polyclonal, 1:2000 dilution; Abcam), and sarcomeric $\alpha$-actinin (mouse monoclonal, 1:500 dilution; Sigma). Coverslips were washed five times with PBS-N and incubated with fluorescein (HDAC1 or HDAC2) and Texas red ( $\alpha$-actinin)-conjugated secondary antibodies (1:500 dilution; Vector Laboratories). Coverslips were washed five times with PBS-N, mounted on glass slides using VectaShield mounting medium with DAPI (Vector Laboratories), and visualized with a fluorescence microscrope.

\section{Western blotting}

Heart and lung tissue was homogenized in lysis buffer $150 \mathrm{mM}$ Tris at $\mathrm{pH} 7.4,150 \mathrm{mM} \mathrm{NaCl}, 1 \%$ Triton X-100, 1 mM EDTA) supplemented with protease inhibitors (Complete Mini, EDTAfree, Roche) and centrifuged at $14,000 \mathrm{~g}$ for $5 \mathrm{~min}$, and supernatant was recovered. Equal amounts were resolved by SDS-PAGE on a $10 \%$ acrylamide gel and analyzed by Western blot using primary antibody for HDAC2 (rabbit polyclonal, 1:5000 dilution; Abcam), followed by goat anti-rabbit IgG HRP-conjugated secondary antibody (Bio-Rad) and detected by enhanced chemiluminescence (Western Blot Luminol Reagent, Santa Cruz Biotechnology). Membranes were reprobed with a primary antibody against eIF5 (rabbit polyclonal, 1:1000 dilution; Santa Cruz Biotechnology) to serve as a loading control.

For detection of $\mathrm{Ca}_{\mathrm{v}} 3.2$, heart tissue was homogenized in lysis buffer (50 mM HEPES at pH 7.5, $250 \mathrm{mM} \mathrm{NaCl}, 0.1 \%$ NP40, $5 \mathrm{mM}$ EDTA/1\% $\beta$-mercaptoethanol) supplemented with protease inhibitors (Complete Mini, EDTA-free, Roche) and centrifuged at $14,000 \mathrm{~g}$ for $3 \mathrm{~min}$, and supernatant was recovered. Equal amounts of protein were resolved by SDS-PAGE on a $4 \%-20 \%$ acrylamide gel and analyzed by Western blot using a primary antibody against $\mathrm{Ca}_{\mathrm{v}} 3.2$ (rabbit polyclonal, 1:500 dilution; Santa Cruz Biotechnology), followed by goat anti-rabbit IgG HRP-conjugated secondary antibody (Bio-Rad) and detected by enhanced chemiluminescence (Western Blot Luminol Reagent, Santa Cruz Biotechnology). Membranes were reprobed with a primary antibody against tubulin (mouse monoclonal, 1:1000 dilution; Sigma) to serve as a loading control.

For detection of troponin isoforms, myofibril preparations were performed as described (Thys et al. 2001). Equal amounts were resolved by SDS-PAGE on a $4 \%-20 \%$ acrylamide gel and analyzed by Western blot using a primary antibody against TnI (goat polyclonal, 1:1000 dilution; Santa Cruz Biotechnology), followed by a donkey anti-goat IgG HRP-conjugated secondary antibody (1:5000 dilution; Santa Cruz Biotechnology), and detected by enhanced chemiluminescence (Western Blot Luminol Reagent, Santa Cruz Biotechnology).

\section{ChIP}

Neonatal rat ventricular myocytes were prepared as described (Antos et al. 2003). Following $24 \mathrm{~h}$ incubation, chromatin was harvested as described (Nelson et al. 2006). Briefly, cells were formaldehyde cross-linked and lysed, and chromatin was sheared by sonication to $~ 500$-base-pair (bp) fragments. Sheared chromatin was immunoprecipitated with antibodies for HA (Sigma), HDAC1 (Abcam), HDAC2 (Abcam), or NRSF (Upstate Biotechnology), and DNA was isolated and analyzed by PCR with primers flanking binding sites for the indicated response element of each gene. Primers are available on request.

\section{Electrocardiography (ECG)}

ECG was performed on sedated neonatal mice using Accutac Diaphoretic ECG Electrodes (ConMed Corp). Mice were al- lowed to adapt to environment prior to recording. Pads were attached to all four limbs, and leads I, II, III, aVR, aVL, and aVF were recorded using PageWriter XLs (Hewlett Packard). Traces were recorded using identical settings between dCKO and wildtype mice $(50 \mathrm{~mm} / \mathrm{sec} ; 2 \mathrm{~mm} / \mathrm{mV})$.

\section{Isoproterenol administration and aortic constriction}

Hypertrophic agonist isoproterenol (Sigma) $(8.8 \mathrm{mg} / \mathrm{kg} / \mathrm{d}$ ) or saline were administered using miniosmotic pumps (model 2001, Alzet) dorsally implanted subcutaneously in 8- to 10-wk-old male mice. Mice were sacrificed $7 \mathrm{~d}$ after isoproterenol infusion, and cardiac hypertrophy was assayed by heart weight, body weight, and tibia length. Cardiac hypertrophy was also induced by pressure overload. Eight-week-old wild-type or HDAC1 $1^{\text {loxP/loxP }} ; \alpha M H C$-Cre male mice underwent a sham operation or were subjected to TAC as described (Hill et al. 2000).

\section{Statistical methods}

Values are presented as \pm SEM unless otherwise noted. Gene expression was normalized to 18S Ribosomal RNA and calculated as relative change. Statistics were calculated with Excel. A $P$-value of $<0.05$ was considered to be statistically significant.

\section{Acknowledgments}

We are grateful to Alisha Tizenor for graphics, and to Nik Munshi for advice on electrophysiology. Supported by grants from the National Institutes of Health, the Donald W. Reynolds Clinical Cardiovascular Research Center, and the Robert A. Welch Foundation to E.N.O. M.H. was supported by a grant from the Deutsche Forschungsgemeinschaft (DFG, HA 3335/21). J.F. was supported by the Muscular Dystrophy Association.

\section{References}

Agah, R., Frenkel, P.A., French, B.A., Michael, L.H., Overbeek, P.A., and Schneider, M.D. 1997. Gene recombination in postmitotic cells. Targeted expression of Cre recombinase provokes cardiac-restricted, site-specific rearrangement in adult ventricular muscle in vivo. J. Clin. Invest. 100: 169-179.

Antos, C.L., McKinsey, T.A., Dreitz, M., Hollingsworth, L.M., Zhang, C.L., Schreiber, K., Rindt, H., Gorczynski, R.J., and Olson, E.N. 2003. Dose-dependent blockade to cardiomyocyte hypertrophy by histone deacetylase inhibitors. J. Biol. Chem. 278: 28930-28937.

Bodor, G.S., Oakeley, A.E., Allen, P.D., Crimmins, D.L., Ladenson, J.H., and Anderson, P.A. 1997. Troponin I phosphorylation in the normal and failing adult human heart. Circulation 96: 1495-1500.

Bolden, J.E., Peart, M.J., and Johnstone, R.W. 2006. Anticancer activities of histone deacetylase inhibitors. Nat. Rev. Drug Discov. 5: 769-784.

Davis, C.A., Haberland, M., Arnold, M.A., Sutherland, L.B., McDonald, O.G., Richardson, J.A., Childs, G., Harris, S., Owens, G.K., and Olson, E.N. 2006. PRISM/PRDM6, a transcriptional repressor that promotes the proliferative gene program in smooth muscle cells. Mol. Cell. Biol. 26: 2626-2636.

Finnin, M.S., Donigian, J.R., Cohen, A., Richon, V.M., Rifkind, R.A., Marks, P.A., Breslow, R., and Pavletich, N.P. 1999. Structures of a histone deacetylase homologue bound to the TSA and SAHA inhibitors. Nature 401: 188-193.

Gao, L., Cueto, M.A., Asselbergs, F., and Atadja, P. 2002. Cloning and functional characterization of HDAC11, a novel member of the human histone deacetylase family. J. Biol. Chem. 277: 25748-25755. 
Gregoretti, I.V., Lee, Y.M., and Goodson, H.V. 2004. Molecular evolution of the histone deacetylase family: Functional implications of phylogenetic analysis. J. Mol. Biol. 338: 17-31.

Grozinger, C.M. and Schreiber, S.L. 2002. Deacetylase enzymes: Biological functions and the use of small-molecule inhibitors. Chem. Biol. 9: 3-16.

Grozinger, C.M., Hassig, C.A., and Schreiber, S.L. 1999. Three proteins define a class of human histone deacetylases related to yeast Hdalp. Proc. Natl. Acad. Sci. 96: 4868-4873.

Hassig, C.A., Tong, J.K., Fleischer, T.C., Owa, T., Grable, P.G., Ayer, D.E., and Schreiber, S.L. 1998. A role for histone deacetylase activity in HDAC1-mediated transcriptional repression. Proc. Natl. Acad. Sci. 95: 3519-3524.

Hill, J.A., Karimi, M., Kutschke, W., Davisson, R.L., Zimmerman, K., Wang, Z., Kerber, R.E., and Weiss, R.M. 2000. Cardiac hypertrophy is not a required compensatory response to short-term pressure overload. Circulation 101: 2863-2869.

Jenuwein, T. and Allis, C.D. 2001. Translating the histone code. Science 293: 1074-1080.

Kee, H.J., Sohn, I.S., Nam, K.I., Park, J.E., Qian, Y.R., Yin, Z., Ahn, Y., Jeong, M.H., Bang, Y.J., Kim, N., et al. 2006. Inhibition of histone deacetylation blocks cardiac hypertrophy induced by angiotensin II infusion and aortic banding. Circulation 113: 51-59.

Khier, H., Bartl, S., Schuettengruber, B., and Seiser, C. 1999. Molecular cloning and characterization of the mouse histone deacetylase 1 gene: Integration of a retrovirus in 129SV mice. Biochim. Biophys. Acta 1489: 365-373.

Kobayashi, T. and Solaro, R.J. 2005. Calcium, thin filaments, and the integrative biology of cardiac contractility. Annu. Rev. Physiol. 67: 39-67.

Kong, Y., Tannous, P., Lu, G., Berenji, K., Rothermel, B.A., Olson, E.N., and Hill, J.A. 2006. Suppression of class I and II histone deacetylases blunts pressure-overload cardiac hypertrophy. Circulation 113: 2579-2588.

Kuwahara, K., Saito, Y., Takano, M., Arai, Y., Yasuno, S., Nakagawa, Y., Takahashi, N., Adachi, Y., Takemura, G., Horie, M., et al. 2003. NRSF regulates the fetal cardiac gene program and maintains normal cardiac structure and function. EMBO J. 22: 6310-6321.

Lagger, G., O'Carroll, D., Rembold, M., Khier, H., Tischler, J., Weitzer, G., Schuettengruber, B., Hauser, C., Brunmeir, R., Jenuwein, T., et al. 2002. Essential function of histone deacetylase 1 in proliferation control and CDK inhibitor repression. EMBO J. 21: 2672-2681.

Lin, H., Yutzey, K.E., and Konieczny, S.F. 1991. Muscle-specific expression of the troponin I gene requires interactions between helix-loop-helix muscle regulatory factors and ubiquitous transcription factors. Mol. Cell. Biol. 11: 267-280.

Maglott, D., Ostell, J., Pruitt, K.D., and Tatusova, T. 2007. Entrez Gene: Gene-centered information at NCBI. Nucleic Acids Res. 35 (Database issue): D26-D31. doi: 10.1093/nar/ gk1993.

Matsuoka, S., Ballif, B.A., Smogorzewska, A., McDonald III, E.R., Hurov, K.E., Luo, J., Bakalarski, C.E., Zhao, Z., Solimini, N., Lerenthal, Y., et al. 2007. ATM and ATR substrate analysis reveals extensive protein networks responsive to DNA damage. Science 316: 1160-1166.

McKinsey, T.A., Zhang, C.L., Lu, J., and Olson, E.N. 2000. Signal-dependent nuclear export of a histone deacetylase regulates muscle differentiation. Nature 408: 106-111.

Minucci, S. and Pelicci, P.G. 2006. Histone deacetylase inhibitors and the promise of epigenetic (and more) treatments for cancer. Nat. Rev. Cancer 6: 38-51.

Nelson, J.D., Denisenko, O., Sova, P., and Bomsztyk, K. 2006. Fast chromatin immunoprecipitation assay. Nucleic Acids
Res. 34: e2. doi: 10.1093/nar/gnj004.

Parmacek, M.S. and Solaro, R.J. 2004. Biology of the troponin complex in cardiac myocytes. Prog. Cardiovasc. Dis. 47: 159-176.

Rodriguez, C.I., Buchholz, F., Galloway, J., Sequerra, R., Kasper, J., Ayala, R., Stewart, A.F., and Dymecki, S.M. 2000. Highefficiency deleter mice show that FLPe is an alternative to Cre-loxP. Nat. Genet. 25: 139-140.

Roopra, A., Sharling, L., Wood, I.C., Briggs, T., Bachfischer, U., Paquette, A.J., and Buckley, N.J. 2000. Transcriptional repression by neuron-restrictive silencer factor is mediated via the Sin3-histone deacetylase complex. Mol. Cell. Biol. 20: 2147-2157.

Roth, S.Y., Denu, J.M., and Allis, C.D. 2001. Histone acetyltransferases. Annu. Rev. Biochem. 70: 81-120.

Sakai, K. and Miyazaki, J. 1997. A transgenic mouse line that retains Cre recombinase activity in mature oocytes irrespective of the cre transgene transmission. Biochem. Biophys. Res. Commun. 237: 318-324.

Sasse, S., Brand, N.J., Kyprianou, P., Dhoot, G.K., Wade, R., Arai, M., Periasamy, M., Yacoub, M.H., and Barton, P.J. 1993. Troponin I gene expression during human cardiac development and in end-stage heart failure. Circ. Res. 72: 932 938.

Shelton, J.M., Lee, M.H., Richardson, J.A., and Patel, S.B. 2000. Microsomal triglyceride transfer protein expression during mouse development. J. Lipid Res. 41: 532-537.

Thomas, P.D., Campbell, M.J., Kejariwal, A., Mi, H., Karlak, B., Daverman, R., Diemer, K., Muruganujan, A., and Narechania, A. 2003. PANTHER: A library of protein families and subfamilies indexed by function. Genome Res. 13: 21292141.

Thomas, P.D., Kejariwal, A., Guo, N., Mi, H., Campbell, M.J., Muruganujan, A., and Lazareva-Ulitsky, B. 2006. Applications for protein sequence-function evolution data: mRNA/ protein expression analysis and coding SNP scoring tools. Nucleic Acids Res. 34 (Web server issue): W645-W650. doi: $10.1093 / \mathrm{nar} / \mathrm{gk} 1229$.

Thys, T.M., Blank, J.M., Coughlin, D.J., and Schachat, F. 2001 Longitudinal variation in muscle protein expression and contraction kinetics of largemouth bass axial muscle. J. Exp. Biol. 204: 4249-4257.

Trivedi, C.M., Luo, Y., Yin, Z., Zhang, M., Zhu, W., Wang, T., Floss, T., Goettlicher, M., Noppinger, P.R., Wurst, W., et al. 2007. Hdac2 regulates the cardiac hypertrophic response by modulating Gsk3 $\beta$ activity. Nat. Med. 13: 324-331.

Verdin, E., Dequiedt, F., and Kasler, H.G. 2003. Class II histone deacetylases: Versatile regulators. Trends Genet. 19: 286293.

Voss, A.K., Thomas, T., and Gruss, P. 1998. Efficiency assessment of the gene trap approach. Dev. Dyn. 212: 171-180.

Xin, M., Davis, C.A., Molkentin, J.D., Lien, C.L., Duncan, S.A., Richardson, J.A., and Olson, E.N. 2006. A threshold of GATA4 and GATA6 expression is required for cardiovascular development. Proc. Natl. Acad. Sci. 103: 11189-11194.

Yang, W.M., Yao, Y.L., Sun, J.M., Davie, J.R., and Seto, E. 1997. Isolation and characterization of cDNAs corresponding to an additional member of the human histone deacetylase gene family. J. Biol. Chem. 272: 28001-28007.

Yasui, K., Niwa, N., Takemura, H., Opthof, T., Muto, T., Horiba, M., Shimizu, A., Lee, J.K., Honjo, H., Kamiya, K., et al. 2005. Pathophysiological significance of T-type $\mathrm{Ca}^{2+}$ channels: Expression of T-type $\mathrm{Ca}^{2+}$ channels in fetal and diseased heart. J. Pharmacol. Sci. 99: 205-210. 


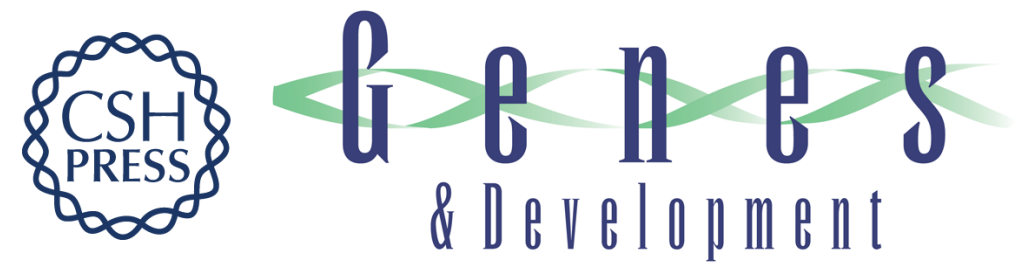

\section{Histone deacetylases 1 and 2 redundantly regulate cardiac morphogenesis, growth, and contractility}

Rusty L. Montgomery, Christopher A. Davis, Matthew J. Potthoff, et al.

Genes Dev. 2007, 21:

Access the most recent version at doi:10.1101/gad.1563807

\section{Supplemental Material \\ http://genesdev.cshlp.org/content/suppl/2007/07/23/21.14.1790.DC1 \\ http://genesdev.cshlp.org/content/suppl/2007/07/10/21.14.1790.DC2}

References

This article cites 41 articles, 20 of which can be accessed free at: http://genesdev.cshlp.org/content/21/14/1790.full.html\#ref-list-1

\section{License}

Email Alerting

Service
Receive free email alerts when new articles cite this article - sign up in the box at the top right corner of the article or click here.

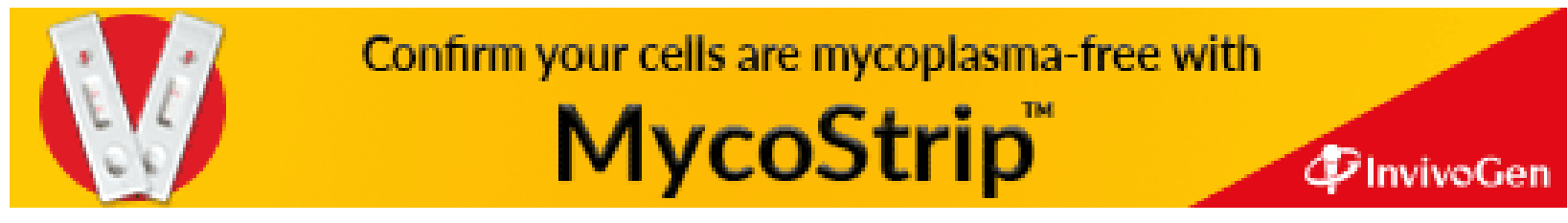

